Artículo de revisión

\title{
Condiciones ambientales y microorganismos adecuados para la obtención de humus de calidad y su efecto en el suelo agrícola
}

\section{ENVIRONMENTAL CONDITIONS AND MICROORGANISMS SUITABLE FOR OBTAINING QUALITY HUMUS AND ITS EFFECT ON THE AGRICULTURAL SOIL}

\author{
GINA LUNA-CANCHARI§*, NICOLE MENDOZA-SOTO§
}

Recibido: 01 octubre de 2020 / Aceptado: 25 noviembre de 2020

\author{
§scuela de Profesional de Ingeniería Ambiental, Facultad de Ingeniería y Arquitectura, \\ Universidad Peruana Unión, Lima, Perú
}

\begin{abstract}
Resumen
Un problema provocado por la sobrepoblación en el Perú y Latinoamérica es el incremento de residuos sólidos, siendo la mala gestión el principal inconveniente. Dentro de las propuestas de solución están el uso de la biotecnologías; como parte de ello, el compostaje donde se emplean organismo como las lombrices, proceso también conocido como vermicompostaje. Existen diversas especies de lombrices que se pueden utilizar en el vermicompostaje; sin embargo, para obtener un humus de calidad, las más eficientes y comercializadas son las lombrices rojas, dentro del grupo, especies resaltantes son la Eusenia andrei y la Eusenia foetida, también llamadas lombrices roja californiana y lombriz tigre. La obtención del humus gracias a estos organismos se da por la síntesis de los residuos orgánicos a nutrientes, ideales para el suelo, proceso que se lleva a cabo en su corto sistema digestivo. Además, para lograr un desarrollo adecuado de las lombrices hay factores ambientales importantes que interactúan como la temperatura, humedad, estructura del sustrato, $\mathrm{pH}$ y conductividad, condiciones ambientales, que se describe en este artículo. Para poder determinar un humus de calidad se debe considerar parámetros tanto físicos-químicos y nutricionales, y no solo basarse en las características organolépticas (color, olor y textura), además un humus de calidad genera impactos positivos al suelo ayudando al desarrollo de la agricultura sostenible.

Palabras clave: Lombrices de tierra, compostaje, condiciones ambientales, calidad del humus
\end{abstract}

\begin{abstract}
A problem caused by overcrowding in Peru and Latin America is the increase in solid waste, poor management being the main drawback. Among the solution proposals are the use of biotechnologies; as part of this, composting where organisms such as worms are used, a process also known as vermicomposting. There are several species of worms that can be used in vermicomposting; however, to obtain a quality humus, the most efficient and commercialized are the red worms, within the group, outstanding species are Eusenia andrei and Eusenia foetida, also called californian red worms and tiger worms. Obtaining humus thanks to these organisms occurs through the synthesis of organic waste into nutrients, ideal for the soil, a process that takes place in its short digestive system. In addition, to achieve a proper development of worms there are important environmental factors that interact such as temperature, humidity, substrate structure, $\mathrm{pH}$ and conductivity, environmental conditions, which are described in this article. In order to determine a quality humus, both physical-chemical and nutritional parameters must be considered, and not only based on the organoleptic characteristics (color, smell and texture), in addition to a quality humus it generates positive impacts on the soil, helping the development of the sustainable agriculture.
\end{abstract}

Keywords:. Earthworms, composting, environmental conditions, humus quality 


\section{INTRODUCCIÓN}

El Perú cuenta con una problemática que la mayoría de países en Latinoamérica lo viven. La sobrepoblación ha traído grandes impactos ambientales, entre ellos el aumento descontrolado de residuos sólidos. Según los informes anuales de residuos sólidos del MINAM (2014), en el Perú se genera 20 mil toneladas de residuos sólidos por día, y que solo Lima metropolitana genera 7400 toneladas por día. Cabe mencionar que más de $50 \%$ de estos residuos producidos son orgánicos, la problemática no hace un enfoque en la producción de residuos si no, en el uso adecuado de estos. Si los residuos no son gestionados convenientemente, pueden generar graves problemas en la conservación del ambiente y a la salud de las personas (Jarauta, 2005). Para el tratamiento de residuos orgánicos existe la posibilidad de implementar sistemas anaerobios (Biogás y Biol) y aerobios (Compost). El presente estudio va relacionado con el proceso de compostaje, que sucede cuando se produce la descomposición de residuos orgánicos por acción de las bacterias, hongos y lombrices en condiciones aerobias (Ministerio del Desarrollo e Inclusión Social, 2014 ).

El humus de lombriz es un abono orgánico que en los últimos años se ha generalizado su uso, sobre todo en la agricultura para el sostenimiento del suelo (Milanés et al., 2005). La lombricultora es un proceso biotecnológico simple. El humus de lombriz como resultado de las transformaciones bioquímicas y microbiológicas que sufre la materia orgánica al pasar por el tracto digestivo de las lombrices, contiene una gran cantidad de microorganismos y nutrimentos, por lo que favorece la estructura y la actividad biológica del suelo, así como la nutrición vegetal (Pérez et al., 2012).

El vermicultivo utilizado como enmienda, permite satisfacer la demanda nutritiva de los cultivos hortícolas en invernadero y reduce significativamente el uso de fertilizantes sintéticos, también se menciona que el uso de este producto tiene un aporte importante de carbono orgánico humificado (Milpa et al., 2012).

Las lombrices son seres vivos capaces de transformar los residuos orgánicos en abonos, este humus, en general, ayuda al suelo teniendo un efecto de mejoramiento en las propiedades físicas, químicas y biológicas, la práctica de la lumbricultura es muy importante para el reciclaje de algunos desechos generados por la agricultura, agroindustria y municipales (Briceño y Pérez, 2017). Sin embargo, aun conociendo las ventajas de incorporar humus de lombriz al suelo, es necesario evaluar su condición, pues de no cumplir con algunos requerimientos de calidad, podría causar efectos adversos en el suelo y las plantas (Melgarejo et al., 1997). Por esa razón, el objetivo de este trabajo fue realizar una revision sobre las condiciones adecuadas para un buen proceso de compostaje producido por lombrices, también se centra en revisar las propiedades que normalmente presenta el humus para determinar su calidad, todo esto enfocado en el mejoramiento del suelo.

\section{LOMBRICES Y VERMICOMPOST}

Domínguez y Gómez (2010), mencionan que hay más de 8000 especies de lombrices registradas y que además existe un promedio de que cada año se descubren 68 especies nuevas, el mismo autor hace referencia a Bouché (1997) pues en su estudio se menciona tres grupos principales de categorización para las lombrices, los tipos de lombrices (Figura 1) descritos son los siguientes: 
- Epígeas: Suelen vivir en la parte superficial de los suelos, alimentándose de la materia orgánica en esa zona del suelo, cuenta con una alta tasa de reproducción y un acelerado metabolismo, además suelen ser especies de tamaño pequeño y raramente forman galerías o madrigueras.

- Endógenas: Habitan en distintas profundidades del suelo, suelen alimentarse de los productos de las raíces o de materia orgánica existente en la capa de tierra, son más longevas, pero tiene una baja tasa de reproducción, además pueden resistir periodos de hambre, y tienden a formar galerías horizontales.

- Anécicas: Suelen moverse constantemente desde la parte superior del suelo hacia distintas profundidades, y en las noches suben a la superficie terrestre para alimentarse de los restos orgánicos, esta circulación permite que las lombrices formen canales verticales los cuales generan aireación en el suelo incrementando la oxigenación del mismo, suelen ser de gran tamaño y su tasa de reproducción suele ser baja.

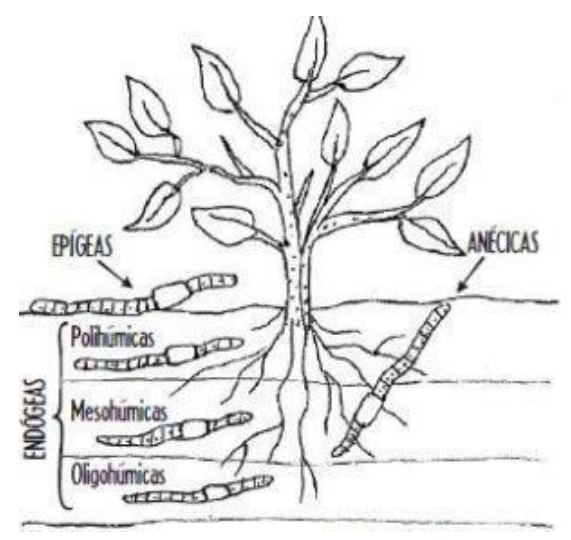

Figura 1. Tipos de lombrices

Sin duda, existen demasiadas especies de lombrices benéficas para el suelo; sin embargo, para la producción de humus, son más eficientes aquellas que contienen una digestión acelerada y de buena asimilación de materia orgánica. Las epígeas, cumplen con esta atribución y son las más empleadas para el vermicompostaje; además cuentan con una alta tasa de reproducción y consumo, como también toleran diversas condiciones ambientales (Domínguez y Gómez, 2010). No obstante, no todas las especies de este tipo de lombriz tiene la misma capacidad de adaptabilidad para ser domesticadas o criadas; por ello, las especies que más resaltan de este grupo son Eisenia spp., Eisenia foetida y Eisenia andrei, también llamadas "lombrices rojas" (Sánchez, 2017), estos organismos son muy parecidos morfológicamente, razón por la que durante muchos años fueron conocidas como una sola lombriz, sin embargo, presentan ciertas diferencias biológicas como la pigmentación del cuerpo, y en especial en la taxología (Domínguez y Pérez, 2010).

Eisenia foetida, presenta colores rojizos y rayas horizontales amarillentas, por lo que comúnmente es llamada "lombriz tigre", y la lombriz Eisenia andrei, es de tonos rojizos uniformes y es conocida como "lombriz roja californiana", no obstante, ambas lombrices pueden convivir en poblaciones mixta con el mismo sustrato (Sánchez, 2017). 
La lombriz más conocida y comercializada es Eisenia foetida, por su corto ciclo reproductivo, debido a que a las 7 u 8 semanas llegan a su madurez sexual, son de mayor longevidad, además son dóciles y adaptables para criar en ambientes reducidos (Díaz, 2002); así mismo, Sánchez (2017) hace referencia a Schuldt (2006) quien señala que esta lombriz actúa con mayor voracidad, cualidad que la convierte en la más solicitada para el vermicompostaje en comparación con la Eisenia andrei.

Domínguez y Gómez (2010) mencionan que existen otras lombrices, que por sus cualidades podrían ser empleadas para el vermicompostaje, estas son, Dendrodrilus rubidus, Dendrobaena veneta, Lumbricus rubellus y Drawida nepalensis.

\section{SISTEMA DIGESTIVO DE LA LOMBRIZ DE TIERRA}

El sistema digestivo de la lombriz roja (Figura 2) inicia por una boca, la cual cumple con la función de succionar el alimento (debido a que carecen de dientes), luego pasa por la faringe donde existen glándulas que generan mucus para lubricar el alimento, en este órgano también se encuentran las fibras musculares adheridas a las paredes, las cuales funcionan como bomba al momento de la succión, seguidamente se conecta al esófago, el cual es de forma tubular, desemboca en el buche, donde los alimentos se almacena de forma temporal, ablandándolos y humedeciéndolos para luego pasar a la molleja, en esta zona los alimentos son masticados y triturados, posteriormente pasa al intestino, donde las grasas, carbohidratos y proteínas son atacados por enzimas digestivas (como la pepsina y la lipasa) y son absorbidos, finalmente lo que no es asimilado es expulsado por el ano (Alas y Alvarenga, 2002; Revera et al., 2017).

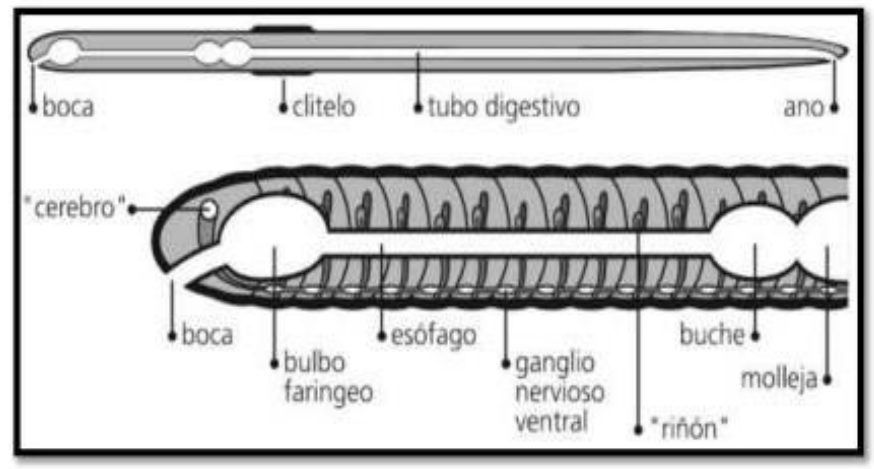

Figura 2. Vista general del sistema digestivo de una Lombriz adulta (Mazariegoz, 2018)

\section{CONDICIONES AMBIENTALES Y ÓPTIMO DESARROLLO DE LA LOMBRIZ}

Domínguez y Gómez (2010) recolectaron una serie de datos y estudios científicos de las condiciones físicas, adecuadas para el desarrollo de las lombrices, los cuales indicaron que existen dos factores fundamentales, estos son:

- Temperatura: El crecimiento es óptimo a $\operatorname{los} 25^{\circ} \mathrm{C}$, pero también pueden desarrollarse entre los 25-28 ${ }^{\circ} \mathrm{C}$, incluso a los $32{ }^{\circ} \mathrm{C}$ siempre en cuando hayan sido aclimatadas 
moderadamente desde el Nacimiento; sin embargo, esta condición inhibe su crecimiento, además, si la temperatura alcanza o supera los $33,3{ }^{\circ} \mathrm{C}$ las lombrices mueren (Domínguez y Gómez, 2010). Por otro lado, las temperaturas mínimas para el crecimiento giran alrededor de los $10{ }^{\circ} \mathrm{C}$, aunque existe estudios que comprueban el crecimiento de las lombrices a temperatura bajo cero; por ello, no es recomendable para el correcto desarrollo (Sánchez, 2017).

- Humedad: Los porcentajes ideales para el desarrollo se encuentran entre 80 y $85 \%$, aunque las lombrices presentan un amplio rango (Domínguez y Gómez, 2010); no obstante, es importante tener el control sobre este factor, si disminuye hasta el $12 \%$ o menos, el proceso de descomposición se ralentiza (Díaz, 2002).

Las lombrices cuentan con una capacidad impresionante con respecto a las condiciones de temperatura y humedad, pues si estas entran en un desequilibrio, ellas pueden activar su estado de inactividad "diapausa", periodo en el cual las lombrices permanecen fuertemente enroscadas hasta que las condiciones ambientales se adecuen (Domínguez y Gómez, 2010).

Sánchez (2017) en su estudio científico sobre el vermicompostaje, señala también otros factores relevantes para el desarrollo óptimo de la lombriz, los cuales son:

- Estructura del sustrato: Es importante que la estructura del sustrato a utilizar permita la difusión pasiva del aire y evite obstruir el desplazamiento de la lombriz, el drenaje del agua y la circulación del oxígeno, este último es el más importante, debido a que las lombrices requieren una concentración de oxigeno de 55\%-65\% (Sánchez, 2017). Este factor también fue mencionado (aireación), por Díaz (2002) él explica que los microorganismos a emplear en el proceso son necesariamente aeróbicos, debido a que los anaeróbicos segregarían metano, ácido sulfúrico y amoniaco, lo cual no es conveniente por los malos olores que causan, así mismo sugirió la remoción periódica de la pila de residuos para apoyar en la aireación.

- pH: Las lombrices presentan un amplio rango en cuanto a pH, siendo de 5 hasta 9 , sin embargo, su función es más eficiente cuando el pH es próximo a la neutralidad (Sánchez, 2017).

- Conductividad eléctrica (CE): Las lombrices rojas tiene una deficiente capacidad para regular las sales en su organismo, razón por la que no pueden vivir en ambientes salinos, pues las mataría, de la misma forma los sustratos a emplear deben contener no más de 8 dSm-1 de conductividad eléctrica, pues generaría la muerte en ellas. (Sánchez, 2017).

Además, la iluminación es factor relevante a tener en consideración puesto que, las lombrices son muy sensibles a los rayos ultravioletas, una ligera exposición a la luz solar podría generarles la muerte, razón por la que es recomendable ubicarlas en zonas de constante sombra (Díaz, 2002). Por otra parte, una condición que también se debe tener en cuenta es la edad del compost, porque si es viejo va a soportar menor densidad de lombrices, y de no tener consideración sobre este factor, puede afectar negativamente a la reproducción de estas (Schuldt et al., 2007). 


\section{CARACTERÍSTICAS PRINCIPALES DEL HUMUS DE CALIDAD}

Por mucho tiempo el humus ha sido considerado como el mejor fertilizante orgánico, porque se puede almacenar durante mucho tiempo sin que sus propiedades se vean alteradas (considerando las condiciones físicas y químicas adecuadas) (Escobar, 2013). Pero lamentablemente en algunos lugares no suelen distribuir un humus de calidad, es decir, suelen ser distribuidos sin cumplir con el requerimiento de madurez, y cuando un humus no está listo para ser aplicado al suelo pueden causar algunos inconvenientes como la inmovilización de nitrógeno, disminuir la concentración de oxígeno o afectar la rizosfora y la germinación de las semillas.

\section{PARÁMETROS DE CALIDAD EN EL HUMUS}

La mayoría de personas y productores, al momento de determinar un humus de calidad suelen considerar aspectos de tipo organoléptico, como el color, olor, y textura al momento de tener tacto con el producto final, pero necesitan un estudio detallado, en donde la calidad de humus está reflejada en las características fisicoquímicas y nutricionales (Melgarejo et al., 1997).

En un estudio realizado por Melgarejo et al. (1997), analizaron cuatro humus con diferente tiempo de compostaje, y de diferente materia prima (H1: Humus de lombriz de pulpa de café con 16 semanas de compostaje ; H2: Humus de lombriz de basuras biodegradables con 14 semanas de compostaje; H3: Humus de lombriz de desechos de cocinas y de huerta con 20 semanas de compostaje; H4: Humus de lombriz de residuos de Rosas con 23 semanas de compostaje) al analizar el contenido de cada uno de ellos concluyeron que el humos que más apto estaba para ser adicionado al suelo fue H4 con 54 . $42 \%$ de materia orgánica, $45.58 \%$ de ceniza y con $3.80 \mathrm{ds} / \mathrm{m}$ Conductividad eléctrica, eso en cuanto a las propiedades fisicoquímicas; en las nutricionales el $\mathrm{H} 4$ presentó elementos como $\mathrm{N}$ total, $\mathrm{P}, \mathrm{K}, \mathrm{Ca}, \mathrm{Mg}$ con mayor porcentaje; en cuento al efecto de la germinación de semillas el H4 presentó 80\%, menor a los demás. Sin embargo, el H3 presentó mayor porcentaje en la germinación con $83 \%$, dando a entender que el humus de lombriz de desechos de cocinas y huerta ayuda más al cultivo; sin embargo, para poder adicionarse al suelo debe tener más tiempo de compostaje, pues en los resultados fisicoquímicos, presentaba menor porcentaje de materia orgánica $(26.35 \%)$, pero en la concentración de ceniza tenía $73.65 \%$ y cabe mencionar que el contenido de materia orgánica correspondía a las pérdidas de calcinación (método usado en esta investigación).

En otra investigación realizada por Salinas et al. (2014) evaluaron la calidad enfocándose en las propiedades químicas del humus de lombriz de rastrojos de tomate y pimentón, después de 90 días, el humus presentaba $2.13 \mathrm{ds} / \mathrm{m}$ Conductividad eléctrica; 37. $9 \%$ de materia orgánica y un $\mathrm{pH}$ de 8.5 , en cuanto al valor nutricional contaba con elementos como nitrógeno, fósforo y potasio.

\section{EFECTO DEL HUMUS EN EL SUELO Y CULTIVOS}

La mejora de la fertilidad del suelo, se ha demostrado mediante investigaciones que al aplicar abonos orgánicos al suelo ayuda en la fertilidad (Konono, 1982), además interviene en la formación de la estructura del suelo y proporciona nutrientes para las plantas y los organismos que dan vida al suelo. 
En una investigación realizada por Zirena y Diaz (1983) informaron que con una aplicación de $200 \mathrm{~g}$ de estiércol humificado, en macetas con $4 \mathrm{~kg}$ de suelo incrementó en un $30 \%$ la obtención de materia seca de sorgo, y también observaron una influencia sobre el desarrollo radicular y una mayor eficiencia en la absorción de fósforo.

Monroy y Viniegra (1981) mencionaron que el afecto de los abonos humificados son: aumento de la capacidad de retención de humedad del suelo a través de su efecto sobre la estructura, porosidad y la densidad aparente, abastecimiento de carbono orgánico como fuente de energía a la flora microbiana heterótrofa y el aumento de la capacidad de intercambio catiónico.

López et al. (2012) realizaron una investigación sobre la evaluación del efecto del humus liquido (humus de Lombriz sólido que puede ser tratado con agua y obtener soluciones acuosas que contienen la concentración de los elementos solubles más importantes presentes en el mismo) aplicándolo en el cultivo de remolacha, pero con diferente método de obtención del humus liquido (decantación, lixiviado y Té de humus), donde el mejor resultado fue el humus del lixiviado (altura del cultivo de remolacha: $55.87 \mathrm{~cm}$; volumen de raíces: 38.75 $\mathrm{cm}^{3}$; peso seco:4.87 g) sin embargo, concluyeron que todos fueron mayor al testigo, donde no hubo aplicación de humus sólido ni líquido, dando a entender que la eficiencia del desarrollo de cultivo se debió a la aplicación del abono, en este caso del humus líquido.

\section{HUMUS EN PERÚ}

La lombricultura es un proceso biotecnológico simple de compostaje y en la actualidad una alternativa importante para lograr la sostenibilidad. Esta tecnología permite la obtención de abono orgánico mediante la descomposición de residuos aprovechables (Iannacone y Alvariño, 2004).

El SENASA (2010) estimó que, una población de 274011 peruanos está involucrados en la agricultura sostenible, 44827 eran agricultores según el Servicio Nacional de Sanidad Agraria y 229184 eran amas de casa, entre los 25 a 44 años.

Vásquez y Iannacone (2014) realizaron una investigación donde evaluaron a 400 personas (120 amas de casas, 40 comerciantes, 80 productores de humus, 40 viveristas y 120 agricultores), determinaron el aporte de la lombricultura para la agricultura sostenible (rural, urbana y periurbana) en el Perú. La encuesta se basó en poder responder las ventajas que tiene el humus de lombriz, como resultado obtuvieron que, los agricultores opinan que la lombricultura presenta un costo alto; los viveristas piensan que mejora el desarrollo de plantones; para los productores de humus, es de fácil producción; para los comerciantes de productos agropecuarios, mejora el desarrollo de plantones; y para las amas de casa, da buen resultado en flores (similar para todas las zonas mencionadas anteriormente), al final concluyeron que la relación entre agricultores, viveristas y amas de casa, con las actividades agrícolas en los ámbitos rural, periurbano y urbano, permite afirmar que la lombricultura aporta significativamente al establecimiento de la agricultura sostenible en el Perú. 


\section{CONCLUSIONES}

De la revisión realizada, se concluye que las lombrices más empleadas para el vermicompostaje son las de tipo epígeas, gracias a su alta taza de reproducción y su acelerada digestión, además la especie que resalta de este grupo es la Lombriz Eisenia foetida, debido a que presenta mayor voracidad y se adapta con mayor facilidad a la crianza doméstica.

Las condiciones ambientales para un buen desarrollo de la lombriz se basan en dos factores fundamentales, la temperatura y la humedad, no obstante, también depende de otros parámetros, como la aireación del sustrato, el pH y la conductividad eléctrica del suelo.

Para poder confirmar que un humus de lombriz es de calidad, según lo investigado el humus debe tener como mínimo un tiempo de compostaje de 90 días, con respecto a la cantidad de materia orgánica debe ser mayor al 50\% usando la metodología de perdida de ceniza por calcinación, también debe presentar elementos como nitrógeno total, potasio, calcio, magnesio y fósforo. Cabe mencionar que debe tener buen color (oscuro), un buen olor y la textura al tocar el producto final debe ser granulada.

Finalmente podemos concluir que al adicionar humus de lombriz al suelo obtenemos muchos beneficios, como la mejora de la estructura, ayuda en la retención de la humedad, incremento de nutrientes, aumento de la actividad microbiana e intensifica la fertilidad del suelo.

\section{Referencias}

Alas, R., \& Alvarenga, A. (2002). Evaluación de sustratos de origen animal y vegetal en la producción de humus y carne de lombriz (Esisenia foetida) (Tesis de pregrado). Universidad de el Salvador

Briceño, A., y Pérez, A. (2017). Utilización del humus Lombriz Roja Californiana (Eisenia foetida) como alternativa amigable al medio ambiente para el cultivo del café (Tesis de pregrado). Universidad Nacional de Nicaragua, Managua.

Díaz, E. (2002). Lombricultura, una alternativa de producción. ADEX.

Domínguez, J., Gómez, B. (2010). Ciclos de vida de las lombrices de tierra aptas para el vermicompostaje. Acta Zoológica Mexicana Número Especial 2: 309-320.

Domínguez, J.; Pérez, M. (2010). Eisenia fetida (savigny, 1826) y eisenia andrei bouché, 1972 son dos especies diferentes de lombrices de tierra. Acta Zoológica Mexicana (nueva serie), núm. 2, 2010, pp. 321-331

Escobar, A. (2013). Usos potenciales del humus (Abono orgánico lixiviado y sólido) en la empresa Fertilombriz. Caldas: Corporación Universitaria La Sallista.

Iannacone, J. \& Alvariño, L. (2004). Eisenia foetida (Savigny, 1826) (Annelida: Lumbricidae) como modelo ecotoxicológico para evaluar lindano y clorpirifos. Acta zoológica Lilloana, 48(1), 5-12.

Jarauta, L. (2005). Digestión anaerobia para el tratamiento de residuos orgánicos: estudio de las necesidades para la implantación en Perú. Energía, 01-02.

López, J., Reyes, V., Reyes, A., y Villa, O. (2012). Evaluación del efecto del humus líquido obtenido 
por tres métodos, en condiciones de maceta y de campo, utilizando maíz (zea mays.l.) y remolacha azucarera (betta vulgaris, $l$ ) respectivamente. Desarrollo Local Sostenible, 5(15), 1-6.

Melgarejo, M., Ballesteras, M., y Bendeck, M. (1997). Evaluación de algunos parámetros fisicoquímicos y nutricionales en humus de lombriz y compost derivados de diferentes sustratos. Revista Colombiana de química, 26(1), 11-19.

Milanés, M., Rodríguez, H., Ramos, R., y Rivera, M. (2005). Efectos del compost vegetal y humus de lombriz en la producción sostenible de capítulos florales en Calendula officinalis L. y Matricaria recutita L. Scielo, 10(1), 1-6.

Milpa, S., González, A., Vásquez, L., y Grenon, G. (2012). Cultivo en maceta de Iris xiphium L. (Iris de Holanda) con diferentes concentraciones de humus de lombriz y sus lixiviados. Facultad de Ciencias Agrarias Uncuyo, 44(2), 109-117.

Ministerio del Desarrollo e Inclusión Social. (2014). Producción y uso de abonos orgánicos: Biol, compost y humus. Lima, Perú: Biblioteca Nacional del Perú.

Monroy, H. y Viniegra, G. (1981). Biotecnología para el aprovechamiento de los desperdicios orgánicos. Ed. A.G.T., México. 260 p

Pérez, Y., Álvarez, J., Mendoza, J., Pat, J., y Gómez, R. (2012). Influencia del humus de lombriz y biofertilizantes en el crecimiento y rendimiento de maíz. Gayana Bot, 69(Nro especial), 15-21.

Konono VA, M.M. 1982. Materia orgánica del suelo- su naturaleza, propiedades y métodos de investigación. Edil. Oikos, Barcelona, España. 365 p

Revera, M., Vargas,E., Gaytán, M., Morales, V. (2017). Estudio de los cambios morfológicos del sistema digestivo de Eisenia foetida en condiciones in vitro en sustratos diferentes. Revista de Ciencias de la Salud. 2017. 4(13): 35-40.

Salinas, F., Sepúlveda, L., y Sepúlveda, G. (2014). Evaluación de la calidad química del humus de lombriz roja californiana (Eisenia foetida) elaborado a partir de cuatro sustratos orgánicos en Arica. Idesia 32(2), 65-99.

Sánchez, J. (2017). Vermicompostaje de residuos orgánicos con lombrices del género Eisenia. Caracterización del producto (tesis de pregrado). Universidad de Sevilla.

Servicio Nacional de Sanidad Agraria (2010). Estadísticas de Producción Orgánica Perú Año 2010. Subdirección de Producción Orgánica. Dirección de Insumos Agropecuarios e Inocuidad Agroalimentaria. Servicio Nacional de Sanidad Agraria - SENASA, Lima.

Schuldt, M., Christiansen, R., Scatturice, L., \& Mayo, J. (2007). Lombricultura. Desarrollo y adaptación a diferentes condiciones de temperatura. REDVET, 1-10.

Vázquez, J., y Iannacone, J. (2014). La lombricultura como aporte para la agricultura sostenible en el Perú. Catedra Villareal, 2(2), 07-20.

Zirena, D.L., y DIAZ, N. T. (1983). Características fertilizantes de afluentes y su utilización como bioabono. Cajamarca, Perú. 81 p. 